\title{
The Patient Voice: At the Intersection of a US Regulatory Revolution
}

\author{
Peter J. Pitts ${ }^{1,2,3}$
}

Published online: 31 August 2016

(C) Springer International Publishing Switzerland 2016

To paraphrase the management guru Peter Drucker, the information revolution will shift from the generation of data to figuring out the meaning and purpose of the data with the patient's perspective in mind. Nowhere is this more pertinent than in the discussion of the future of the patient voice, real world evidence [patient outcomes data, not clinical endpoints that may or may not be important to patients, such as glycated hemoglobin (HbA1c), cholesterol levels, or blood pressure], personalized medicine and the role of the US Food and Drug Administration (FDA).

A key insight comes from the former Director of the US Agency for Healthcare Research and Quality (AHRQ) J.M. Eisenberg who suggests, "Globalize the evidence, localize the decision."

At the FDA, the advancement of regulatory science (such as the incorporation of patient centered/real world data), much depends on willingness and ability to implement new regulatory approaches based on infrastructure, capabilities, and trust between stakeholders. The end goal is the same for all: ensuring optimal use of resources for healthcare systems; improving access to value-adding medicines for patients; and appropriate reward for innovation. It's time to address these issues head on.

Peter J. Pitts

ppitts@cmpi.org

1 Center for Medicine in the Public Interest, New York, NY, USA

2 École Supérieure des Sciences Économiques et Commerciales, Paris, France

3 École Supérieure des Sciences Économiques et Commerciales, Singapore, Singapore
The FDA is taking this challenge to heart-and in writing. Per the FDA's Prescription Drug User Fee Act (PDUFA) VI "commitment letter," [1] the agency will face some real world deadlines to advance the use of real world evidence. But, since we're dealing with the real world, let's get real-guidance is unlikely until the end of 2022 at the earliest. (That's the timeline agreed to via the PDUFA VI negotiations.)

Step 1 towards these new 21 st century rules of the regulatory road will be a series of public meetings and regulatory workshops. It will be curious to see who shows up at the table.

According to the FDA, a key benefit of PDUFA VI is to facilitate the systematic integration of the patient perspective into the development and approval process of new medicines, including the use of patient-reported outcomes (PROs) [2].

The agency has described factors it would consider when evaluating the relevance, reliability and quality of real world evidence, and also suggests when it might use such data to make decisions about medical devices.

To evaluate the reliability of data, the FDA will assess how the data were collected, their adequacy for answering relevant questions, and whether they were collected in a manner that minimizes bias. The guidance says a prospective protocol is "essential to ensure reliability" of real world evidence. The guidance says the FDA might use such evidence to expand a device's approved indications, for postmarket surveillance, and as a control for studies of subsequent devices [3].

\section{Nothing About Me Without Me}

According to a recent white paper from the Network for Excellence in Health Innovation (NEHI) [4]: 
Individuals and groups who are not trained in data analysis face a different challenge. Transparency policies at the NIH, FDA, and other agencies may guarantee access to data and analyses, but does not necessarily equip all stakeholders to review studies in a meaningful way.

RWE [real world evidence] and RWD [real world data] are based in large part on personal data collected in routine patient-clinician encounters - granted. As a result, many RWE proponents believe research grant makers have an obligation to support patients and patient organizations in developing their own capability to conduct meaningful reviews of RWE, particularly when important health care or health policy decisions are made on the basis of RWE. The principle of RWE will play a limited role in future health care decision-making unless transparency standards are applied. The principle of "nothing about me, without me" will loom larger as RWE becomes a greater factor in decision-making about health care.

PCORI [Patient-Centered Outcomes Research Institute] has created a foundation for this style of engagement in the research process and further capacity needs to be developed among patient groups to assess and use the evidence.

\section{Access to Experimental Medicines}

Expanded access to experimental medicines is another area where the patient voice and FDA policy meet-and not always harmoniously. In January 2008, the US Supreme Court, without comment, opted not to accept an appeal of Abigail Alliance v. von Eschenbach [5]. In other words, the federal appeals court ruling that patients do not have a constitutional right to experimental drugs stands.

This is a tough, emotional issue and, with such heated rhetoric on both sides, it's easy to lose sight of the fact that everyone wants the same thing-expanded access to drugs under clinical investigation.

Perhaps, as part of the FDA's current initiatives to enhance both the timeliness and weight of the patient voice, sponsor development of expanded access protocols should involve patient organizations from the very outset. We have to take into serious strategic and tactical consideration the phenomenon of social media and the pressures it can (and does) bring to bear on both sponsors and the FDA. Consider the FDA's reintroduction of the previously recalled multiple sclerosis treatment Tysabri [6]. The agency's reexamination of the drug was due as much to sponsor communications as it was to the outcry of the patient community [7]. Maybe it's time to harness that power to make the process for both protocol development and access both more inclusive and better.

Expanded access is a highly contentious issue-and for all the wrong reasons. Four US states (AZ, CO, MO, LA and MI) now have so-called "right to try legislation," whereby doctors covered by the laws can bypass the FDA and go directly to drug makers to discuss the risks and (potential) benefits of unapproved drugs in clinical development. They can then decide with their patients whether they will request access.

The "right to try" albatross can trace its philosophical roots to a policy paper from the Goldwater Institute, titled, "Everyone Deserves the Right to Try: Empowering the Terminally Ill to Take Control of their Treatment" [8]; it points the finger at the FDA as a roadblock to access. Nothing could be further from the truth.

Between October 2013 and September 2014, the FDA received 1882 requests for expanded access to investigational new drugs (INDs) and treatment protocols-and approved 1873 of them. That's an outstanding batting average by any standard. It also puts into perspective the Goldwater Institute's claim that "over half a million cancer patients and thousands of patients with other terminal illnesses die each year as the bureaucratic wheels at the FDA slowly turn." [8] What the Goldwater paper presents is a libertarian platform- "The burdens imposed on a terminal patient who fights to save his or her own life are a violation of personal liberty." Maybe so, but the Supreme Court, as already mentioned, has ruled otherwise. Alacrity is important, certainly. But process is important too, as is collecting data on expanded access use.

The Goldwater paper does raise important questions, such as at what point in the drug development process should an investigational product be available to patients. The paper argues for phase I. That's an aggressive position, but one worth debating.

One item that the paper ignores is that for the FDA to address single patient INDs with both more careful attention and speed, a huge increase in funding would be required. That's more than the 800 -pound gorilla sitting in the room-it's the 800-pound gorilla sitting on the chests of desperately ill patients who want access to investigational medicines. The current figure of 1882 requests is almost exactly double the number from only a year earlier. Swifter regulatory turn-around time requires more staff.

The author quotes Patty Delaney. Patty (who passed away in 2008) was the FDA's main liaison to the cancer community and a tireless soldier for "doing the right thing." She was a pit bull on behalf of patients.

According to the paper, "As Patty Delaney, the former director of the FDA's cancer liaison program explained in 
2007, the patient has a right to be heard, but in the end, it's the data that matters. FDA opinions about safety and efficacy are always based on data."

I'll side with Patty. There has to be oversight. There has to be a responsible body that sets rules. Libertarianism, when it comes to pharmaceutical safety, isn't in the best interests of public health. Does that mean the status quo works? No, but expanded access to experimental drugs simply can't and shouldn't morph into total, unfettered access. That doesn't mean the status quo is working. What it means is that the FDA needs to figure out a way to dramatically broaden and facilitate expanded access to experimental drugs under its review. And everyone concerned needs to keep up the pressure to improve the current system.

\section{Who Pays for Access?}

Another issue that remains at large is who pays for access to these unapproved drugs. Sometimes the drug company will bare all costs, other times some costs, and just as often it's the patient who bears the cost. And while the price of an unapproved product is regulated (via draft guidance), Institutional Review Board (IRB) and other related costs are often borne by the patient. There's a role for the Federal government here. (How about a Federal fund that pays for access for any approved FDA expanded access IND or protocol?)

All sides want the same thing-expedited expanded access programs. But name calling and bridge burning doesn't bring anyone closer together or experimental drugs to dying patients any faster. Let's expedite access by enlarging the expanded access ecosystem.

When it comes to the patient voice (or any voice), the plural of anecdote isn't data. But the plural of data is science.

\section{Patient Focused Drug Development}

The Food and Drug Administration Safety and Innovation Act (FDASIA) enshrines the concept of patient-focused drug development. Per the FDA's own website [9], "Patients who live with a disease have a direct stake in the outcomes of the drug review process and are in a unique position to contribute to the entire medical product development enterprise. Under FDASIA, the FDA will increase patient participation in medical product regulation".

The PDUFA V agreement provides for a new process enhancement under a commitment that will provide a more systematic and expansive approach to obtaining the patient perspective on disease severity or the unmet medical need in a therapeutic area to benefit the drug review process. In other words, the patient perspective will provide context in which regulatory decision-making is made, specifically the analysis of the severity of the condition and the current state of the treatment armamentarium for a given disease.

But patient input needs to be more than anecdotal; it needs to be data driven so that decisions can be more scientifically driven by the patient community. And nowhere is that more urgently needed than in discussions over risk/benefit calculations.

Steve Usdin's cover story in BioCentury, "Calming the Pendulum," makes the case in the first sentence: "Regulators and drug developers have converged on the idea that enhancing and broadening patient engagement is a key to improving drug development and adjudicating controversies over benefit-risk decisions and the value of medicines." [10]

Absolutely right. But that's not patient-focused drug development (after all, isn't all drug development patient focused?); it's patient-driven drug development (PD3). It's time to change the name to properly fit the task. PD3 places the patient voice squarely in the middle of the drug development ecosystem.

Usdin quotes Dr. John Bridges (senior fellow at the Center for Medicine in the Public Interest and Associate Professor at the Johns Hopkins Bloomberg School of Public Health), who says that the FDA's use of decisionbased preference data "changed the world." If only it were that easy.

Consider the 21st Century Cures Act currently pending in Congress. Its philosophy is to make the FDA an accelerator rather than a sea-anchor to getting life-saving new medicines to desperately ill patients. Usdin writes, "Patient engagement provisions in the 21st Century Cures discussion draft [11] were written based on input from patient groups, industry and FDA. They are intended to create regulatory certainty around the use of patient perspectives, including formal pathways for patient groups and companies to submit information about patient preferences, as well as defining how FDA will incorporate these submissions into approval and other decisions."

In Bridges' paper, "Identifying the Benefits and Risks of Emerging Treatments for Idiopathic Pulmonary Fibrosis: A Qualitative Study," [12] he provides important qualitative evidence on stakeholders' views as to important issues associated with emerging therapies for idiopathic pulmonary fibrosis.

Bridges et al. identify multiple issues spanning the impact of emerging therapies, including the need to document the patient experience with treatment, and factors associated with disease progression.

The paper discusses the value of qualitative research both in understanding the benefits and risks of emerging 
therapies and in promoting patient-centered drug development.

\section{Patient-Reported Outcomes}

What about PROs? These are any report of the status of a patient's health condition coming directly from the patient, without interpretation by physicians or anyone else, about how the patient functions or feels in relation to a health condition and its treatment. Clearly, this is an important way to recognize "patient voice" input, but from the FDA perspective, is such information well-specified and reliable; specific for an identified target population; specific for a target indication; adequately validated with measurement properties?

To further advance its Patient-Focused Drug Development program, the FDA could pursue intramural, qualitative research to establish PRO content validity. This might include:

- Focus groups to generate a pool of patient outcomerelated domains and their components.

- The symptoms and functions or activities impacted by disease that are most important to patients.

- Surveys including a larger and more diverse sample of patients with a given condition. (For example, examine the importance and relevance of domains identified by literature review, expert opinion or among a smaller set of patients, to validate these items and perhaps explore other measurement characteristics.) [13]

\section{Concluding Thoughts}

Patient passion is important to share. When combined with data and a more dispassionate understanding of regulatory paradigms, a patient-driven pathway can, and must, evolve into a tool used to impact regulatory decision-making.

As FDA Commissioner Rob Califf and Deputy Commissioner Rachel Sherman recently commented:

Creating knowledge requires the application of proven analytical methods and techniques to biomedical data in order to produce reliable conclusions... There must be a common approach to how data is presented, reported and analyzed and strict methods for ensuring patient privacy and data security... Rules of engagement must be transparent and developed through a process that builds consensus across the relevant ecosystem and its stakeholders... To ensure support across a diverse ecosystem that often includes competing priorities and incentives, the system's output must be intended for the public good and be readily accessible to all stakeholders [14].

We need to develop proposals that modernize the information used in the evaluation of the value of treatments. Just as the key scientific insights guiding the FDA Critical Path program are genetic variations and biomedical informatics that predict and inform individual responses to treatment, we must establish a science-based process that incorporates the knowledge and tools of personalized medicine in reimbursement decisions: true evidence-based, patient-centric medicine.

Today, right now, we need a Critical Path for Healthcare Technology Assessment (HTA) to begin the process of developing a similar list of ways new discoveries and tools (such as electronic patient records) can be used to improve the predictive and prospective nature of clinical outcomes. In an era of personalized medicine, one-size-fits-all treatments and reimbursement strategies are dangerously outdated. Accepting real world evidence does not mean discarding the randomized "gold standard"-it means augmenting it.

It's a complicated proposition, but such a goal is as simple as it is essential; cost must never be allowed to trump care, and short-term savings must not be allowed to trump long-term outcomes. Just as we need new and better tools for drug development, so too do we need them for HTA.

An HTA model for the 21st century should reflect and measure individual response to treatment based on the combination of genetic, clinical, and demographic factors that indicate what keep people healthy, improve their health, and prevent disease. A rapidly aging society demands a new healthcare paradigm capable of providing for its needs in the 21st century. Equality of care must be matched with quality of care.

When it comes to the regulatory science of real world evidence, we are still in early days, but the times they are a changin'.

\section{References}

1. http://www.fda.gov/downloads/ForIndustry/UserFees/Prescription DrugUserFee/UCM511438.pdft. Accessed 20 Aug 2016.

2. http://phrma.org/sites/default/files/pdf/Factsheet-PDUFA-VI.pdf. Accessed 17 Aug 2016.

3. http://www.fda.gov/downloads/MedicalDevices/DeviceRegulation andGuidance/GuidanceDocuments/UCM513027.pdf. Accessed 18 Aug 2016.

4. http://www.nehi.net/writable/publication_files/file/nehi_2016_ rwe_statement.pdf. Accessed 20 Aug 2016.

5. https://scholar.google.com/scholar_case?case $=129640372976772$ 2899\&q=Abigail+Alliance+v.+von+Eschenbach.\&hl=en\&as sdt=6,33\&as_vis=1. Accessed 19 Aug 2016. 
6. http://www.fda.gov/NewsEvents/Newsroom/PressAnnouncements/ 2006/ucm108662.htm. Accessed 21 Aug 2016.

7. http://www.jmir.org/2014/2/e13/?trendmd-shared $=0$. Accessed 17 Aug 2016.

8. http://goldwaterinstitute.org/en/work/topics/healthcare/right-to-try/ everyone-deserves-right-try-empowering-terminally-/. Accessed 18 Aug 2016.

9. http://www.fda.gov/RegulatoryInformation/Legislation/Significant AmendmentstotheFDCAct/FDASIA/ucm 311045.htm. Accessed 20 Aug 2016.

10. http://www.biocentury.com/biotech-pharma-news/coverstory/201502-23/what-industry-fda-must-do-to-realize-the-potential-ofpatient-engagement-a01?utm_source=eTOC\&utm_medium=email
\&utm_campaign=WeeklyTOC\&utm_content=link. Accessed 19 Aug 2016.

11. https://energycommerce.house.gov/cures. Accessed 18 Aug 2016.

12. Bridges JFP, Paly VF, Barker E, et al. Identifying the benefits and risks of emerging treatments for idiopathic pulmonary fibrosis: a qualitative study. Patient. 8:85. doi:10.1007/s40271-014-0081-0.

13. http://www.fda.gov/downloads/AboutFDA/CentersOffices/Office ofMedicalProductsandTobacco/CDER/UCM310754.pdf. Accessed 20 Aug 2016.

14. http://blogs.fda.gov/fdavoice/index.php/2016/05/what-we-meanwhen-we-talk-about-evgen-part-ii-building-out-a-national-systemfor-evidence-generation/. Accessed 17 Aug 2016. 